\title{
Clinical application and accuracy analysis of 3D printing guide plate based on polylactic acid in mandible reconstruction with fibula flap
}

\author{
Po Wu ${ }^{1 \#}$, Lin $\mathrm{Hu}^{2 \#}$, Hao $\mathrm{Li}^{1}$, Lin Feng ${ }^{4}$, Yu Liu ${ }^{1}$, Shuo Zhang ${ }^{4}$, Xue-Chao $\mathrm{Li}^{3}$, Mei-Ling Zhang ${ }^{5}$, \\ Shu-Yong Yang ${ }^{1}$, Rong-Jian $\mathrm{Lu}^{3}$ \\ ${ }^{1}$ Department of Stomatology, The General Hospital of Western Theater Command PLA, Chengdu, China; ${ }^{2}$ Department of Stomatology, Beijing \\ Chaoyang Hospital, Beijing, China; ${ }^{3}$ Department of Stomatology, Fifth Medical Center of Chinese PLA General Hospital, Beijing, China; ${ }^{4}$ Institute \\ of Stomatology \& Oral Maxilla Facial Key Laboratory, Chinese PLA General Hospital, Beijing, China; ${ }^{5}$ Department of Stomatology, Beijing \\ Huairou Hospital of Traditional Chinese Medicine, Beijing, China \\ Contributions: (I) Conception and design: P Wu, L Hu; (II) Administrative support: RJ Lu, SY Yang; (III) Provision of study materials or patients: \\ H Li, L Feng; (IV) Collection and assembly of data: Y Liu, S Zhang; (V) Data analysis and interpretation: XC Li, ML Zhang; (VI) Manuscript \\ writing: All authors; (VII) Final approval of manuscript: All authors. \\ "These authors contributed equally to this work. \\ Correspondence to: Rong-Jian Lu. No. 8 Dongda Street, Fengtai District, Beijing, China. Email: lujian9806@sohu.com; Shu-Yong Yang. The General \\ Hospital of Western Theater Command PLA, Chengdu, China. Email: doctoryangsy@hotmail.com.
}

Background: Due to the special anatomy morphology and physiological function of the mandible, it has always become a challenge to accurately reconstruct the mandibular defect in maxillofacial surgery. Digital three dimensions (3D) printing surgical guide, as the effective method for individual and accurate surgery, is a hotspot of clinical research at present. In this study, 3D printing PLA surgical guide plate was applied to reconstruct the mandibular defect with fibula flap, its clinical effect and accuracy were evaluated, which aimed to improve the accurate reconstruction of mandibular defects.

Methods: After sterilization, the dimension deformation of the PLA standard specimen were measured. Eighteen patients diagnose with mandibular tumor were collected as observation objects. Then partial mandible resection and simultaneous mandible reconstruction with fibula graft were implemented according to the computer-aided design plan. The clinical effects of 3D printing PLA guide plates application were evaluated by facial contours, occlusal stability and chewing function. Through registering the postoperative computed images reconstruction with preoperative designed shape, the reconstruction accuracy was evaluated by detecting the maximum difference including the distance between lateral convex point of the condyles, the distance between medial convex point of the condyles and the horizontal contained angle between long axis of the condyles.

Results: After high temperature steam sterilization, the curvature of the PLA specimen with 100\% filling rate and $4.8 \mathrm{~mm}$ thickness were the smallest and their dimension deformation had no statistical significance $(\mathrm{P}>0.05)$. The minimally deformed 3D printing PLA guide plate were smoothly placed in the right place during the operation. After surgery, the face was symmetrical, the occlusal relationship was restored well and no deviation of the mandibular movement were found. The spiral computed tomography (SCT) scanning showed that the distance between lateral/medial convex points of the condyle and the horizontal contained angle were $128.34 \pm 8.68 \mathrm{~mm}, 88.69 \pm 6.75 \mathrm{~mm}$ and $145.87^{\circ} \pm 12.01^{\circ}$. Compared with preoperative design, the maximum deviation of the actual postoperative registration was $1.67 \pm 0.63$, and the difference was not statistically significant $(\mathrm{P}>0.05)$.

Conclusions: The application of 3D printing PLA guide plate in the segmental section and reconstruction of the mandible can effectively simplify the operation, and better reconstruct the continuity of the mandible. The surgical accuracy can fully meet clinical needs with relatively low prices.

Keywords: 3D printing guide plate; polylactic acid; mandibular reconstruction; accuracy 
Submitted Sep 08, 2020. Accepted for publication Feb 26, 2021.

doi: 10.21037/atm-20-6781

View this article at: http://dx.doi.org/10.21037/atm-20-6781

\section{Introduction}

Mandible is the most important bone scaffold of the lower face that not only support the facial contours, but also maintain chewing, phonation and other oral functions (1). Mandibular defects, especially segmental defect, resulted from trauma, infection or tumor resection will cause functional incapacitation and facial deformity, which seriously affect the living quality and mental health of patients $(2,3)$. Over recent years, there is greater demand for the postoperative recovery of appearance and occlusal functional. How to achieve personalized and accurate reconstruction of mandible defects becomes the hot spot of clinical research.

Free fibular flap transplantation is one of the best ways to reconstruct mandibular segmental defects. Unfortunately, traditional operation mode mostly depends on the surgeon's clinical experience, which hardly satisfies the requirement of accurate reconstruction. In addition, traditional surgery has some shortcomings, such as time-consuming, more intraoperative bleeding and unsatisfactory functional restoration of the mandible (4).

With the development of digital technology and the higher requirement of surgical precision, the application of $3 \mathrm{D}$ guide plate in accurate reconstruction for bone defects has gained more attention. Through 3D printing guide plate, preoperative surgical design was able to be precisely duplicated into mandibular reconstruction surgery, which could effectively improve the surgical accuracy, reduce the difficulty of fine surgery, shorten the operation time (5).

Although photosensitive resin and metal printing guide plate commonly used in clinical have high forming accuracy $(6,7)$, the expensive raw materials and supporting equipment, and the high cost of maintenance limits its clinical wide-ranging application. PLA is a kind of degradable polymer with good biocompatibility and high strength, which has been approved as human implant by US food and drug administration (FDA)and conventional materials for $3 \mathrm{D}$ printing (8). However, the clinical application and accuracy of $3 \mathrm{D}$ printing surgical guide plate based on PLA are still rarely reported.

This research intended to analyze the influence of highpressure steam sterilization on the deformation of the $3 \mathrm{D}$ printing PLA guide and prepare mandibular osteotomy guide plate. According to the computer-aided design plan, the mandibular defect after tumor resection was reconstructed under the guidance of $3 \mathrm{D}$ printing PLA guide plate. The difference of postoperative morphology between the actual and the simulated operation, and threedimensional migration of the mandible were measured to investigate the feasibility of $3 \mathrm{D}$ printing PLA guide plate in mandible reconstruction.

We present the following article in accordance with the MDAR reporting checklist (available at http://dx.doi. org/10.21037/atm-20-6781).

\section{Methods}

\section{Main materials and instruments}

Polylactic acid $\left(M_{W}=1 \times 10^{5} \mathrm{~g} / \mathrm{mol}\right.$; Shanghai Yisheng Industrial Co., Ltd., China), a fused deposition modeling (FDM) 3D printer (BEGO, Germany), a vacuum sterilizer (MAST-A-1200; Xinhua Medical Instruments Co., Ltd., China), Mimics software 15.0 (Materialise, Belgium), Solidworks 14.0 (solidworks, USA), AutoCAD 3D design software (Autodesk, USA), a 3D dental scanner (TRIOS INTRAORAL SCANNERS, 3Shape, Denmark), Open source software KISSlicer, and a piral CT 64-slice scanner (Philips, Netherlands). All of the above devices were used in this study.

The FDM 3D printer parameters: positioning accuracy: $\mathrm{X} / \mathrm{Y}$ axis $=0.011 \mathrm{~mm}, \mathrm{Z}$ axis $=0.0025 \mathrm{~mm}$; printing accuracy $=0.1-0.2 \mathrm{~mm} / 100 \mathrm{~mm}$; printing layer thickness $=0.1-0.2 \mathrm{~mm}$; nozzle diameter $=0.4 \mathrm{~mm}$; printing speed $=25 \mathrm{~mm} / \mathrm{s}$; filament diameter $=2 \mathrm{~mm}$; The vacuum sterilizer parameters: working nozzle temperature $=200{ }^{\circ} \mathrm{C}$; working temperature of the bottom plate $=30^{\circ} \mathrm{C}$.

PLA guide plates were sterilized at a temperature of $134^{\circ} \mathrm{C}$ for 600 seconds, with the internal pressure limit of $210 \mathrm{kPa}$, followed by drying for 780 seconds.

\section{Deformation detection by PLA guide plates with different filling rates after high temperature sterilization}

According to the ASTM D790-10 (Standard Test Methods for Flexural Properties of Unreinforced and Reinforced Plastics and Electrical Insulating Materials) (9), Solidworks 


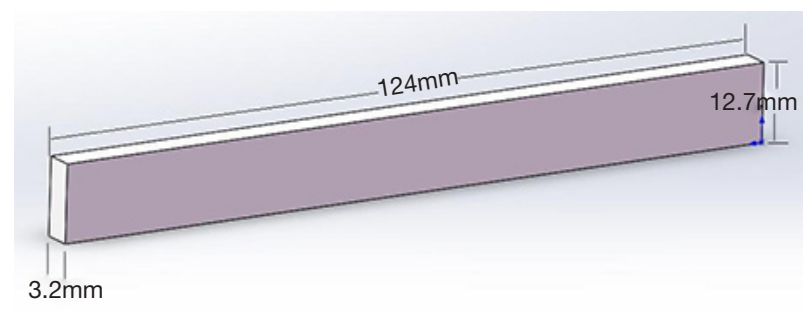

Figure 1 Dimensions of the standard test pieces.

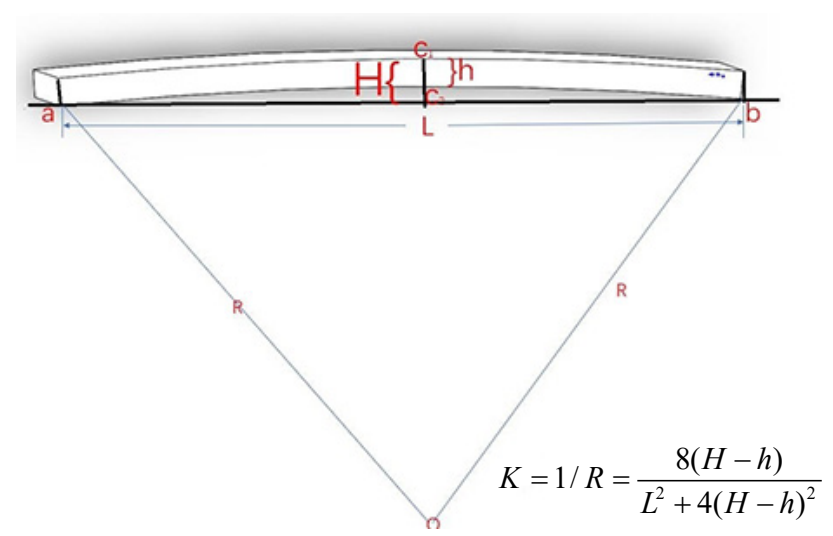

Figure 2 Schematic diagram of the curvature calculation of test pieces after high temperature sterilization.

14.0 software was used to design the standard samples (Figure 1) and generate the STL file, which was imported into the slicing software, KISSlicer, for common printing parameter settings (skin thickness $1 \mathrm{~mm}$, number of loops 3, extrusion width $0.45 \mathrm{~mm}$, infill extrusion width $0.45 \mathrm{~mm}$, layer thickness $0.2 \mathrm{~mm}$ ). The filling rate of samples are was set t $20 \%, 50 \%$, and $100 \%$ in order to generate a print file in .gcode format, which was imported into an FDM 3D printer for printing to complete standard samples. Samples were then individually placed in a sterilizer at high temperature, and high-pressure sterilized. After the autoclave sterilization pressure reaches $1,250 \mathrm{kPa}$, the sterilization chemical indicator card changes color, indicating that the sample reaching the sterilization conditions, then naturally cooled. They were placed on the horizontal surface with the convex surface of the curved test piece facing upwards. To acquire measurements for comparison, a Vernier caliper was used to measure the thickness $(\mathrm{a}, \mathrm{b})$ at both ends of the samples before and after high temperature sterilization, as well as the thickness (h) at the midpoint (c), the length of the $\mathrm{a}-\mathrm{b}$ connection line $(\mathrm{L})$, and the height from the midpoint to the water platform $(\mathrm{H})$. Sterilized samples were approximated as a segment of a circular arc, then calculate the curvature of the $\operatorname{arc~K}$ (Figure 2).

\section{Deformation detection of PLA guide plates with different thicknesses after high temperature sterilization}

According to the process described above, the filling rate with the smallest deformation was selected, and the thickness of the PLA guide plate samples with the same length and width were prepared to be thickness was 3.2, 4.0 and $4.8 \mathrm{~mm}$ respectively. The deformation in each sample before and after high temperature sterilization was measured by Vernier calipers.

\section{Design and production of personalized 3D-printed PLA surgical guide plates}

Spiral computed tomography (SCT) scan of the maxillofacial region and donor calf were conducted before the operation. The scanning images were imported into Mimics 17.0 software for $3 \mathrm{D}$ reconstruction, which was used to simulate the tumor resection and determine the cutting plane, surgical path, osteotomy thickness, etc. With the help of the original mandible or mirror image technique, the affected mandible was reconstructed with reference to the unaffected side (Figure 3). 3D data of the mandible and fibula were imported into the software design guide in STL format, and then sent to the $3 \mathrm{D}$ printer for printing and completion of the PLA surgical guide plate.

\section{Subjects}

From May 2018 to October 2019, 18 patients diagnosed with segmental bone defect after mandible tumor surgery in the General Hospital of Western Theater Command were selected. All patients, with an average age of $40.2 \pm 4.7$ years, underwent fibular myocutaneous flap grafting to have the mandibular defect reconstructed with assistance from a 3D-printed PLA guide plate.

The inclusion criteria for patients were the following: (I) patients diagnosed with mandibular tumor needing to undergo partial resection of mandible; (II) patients with other invading of malignant tumors requiring partial resection of the mandible; (III) patients without contraindications for surgery and anesthesia; (IV) patients who voluntarily joined this study with informed consent.

The exclusion criteria were the following: (I) patients with inoperable oral malignant tumor owing to systemic or 

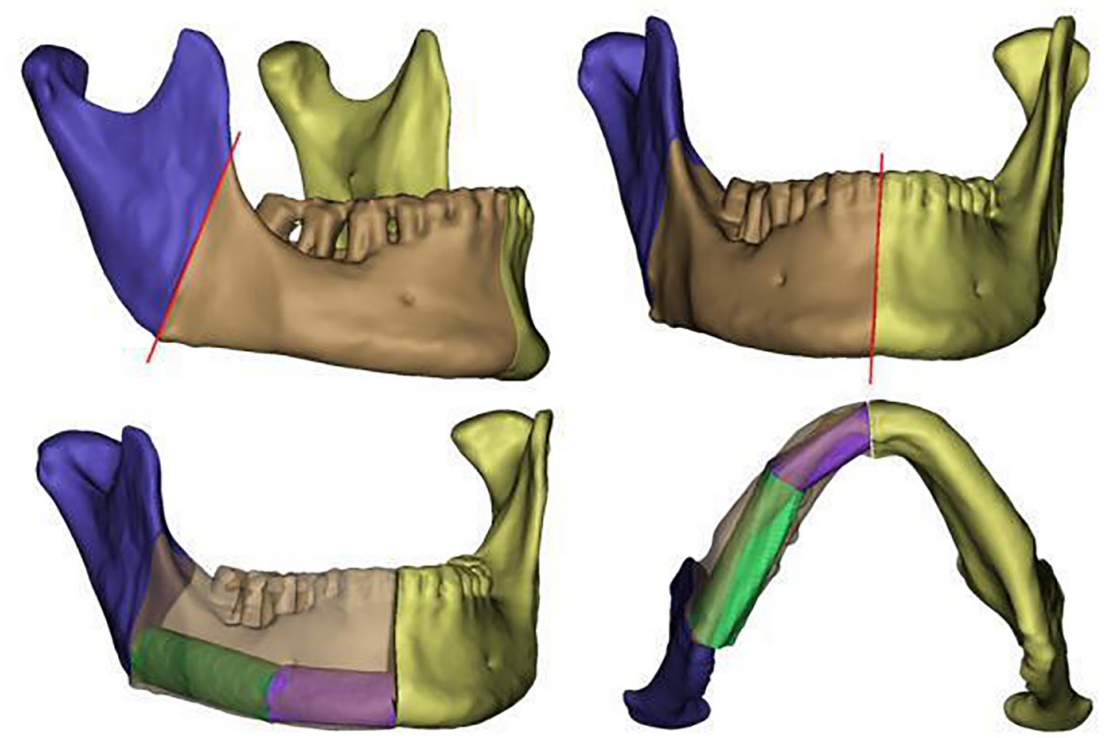

Figure 3 Osteotomy simulation and mandibular reconstruction by Mimics 17.0 software.

extensive metastasis; (II) patients with other severe systemic diseases; (III) patients with obvious organ dysfunction.

All procedures performed in this study involving human participants were in accordance with the Declaration of Helsinki (as revised in 2013). This study was approved by the Medical Ethics Committee of the General Hospital of the Western Theater command of the PLA (No. 2018-5-15). Informed consent was obtained from all the patients.

\section{Operation process}

According to the pathological changes, the surgical incision was designed to expose the mandibular lesions. Based on the preoperative design, 3D-printed osteotomy guide plates were used to resect the mandibular lesions, and the free fibular flap was prepared and shaped. Then, the shaped fibular muscle flap was successfully placed under the guidance of the preformed titanium plate. After vascular anastomosis, the fibula was fixed with titanium screws to complete the reconstruction of the mandibular defects. Postoperative routine prophylactic use of antibiotics and wound dressing was implemented.

\section{Observation index}

Clinical indicators including facial shape, occlusal stability, mouth opening degree, chewing, and phonation function were used to evaluate the clinical efficacy under the following system: grade I, good recovery of contralateral facial appearance; grade II, good phonation and masticatory function, but slight depression on the face and poor bilateral symmetry; grade III, poor phonation function and masticatory function, and obvious concavity on the affected side, with poor bilateral symmetry; grade IV, presence of infection, bone graft exposure, and other phenomena resulting from prosthesis removal. Grade I or II were considered as good recovery, and grade III and IV were considered recovery.

\section{Measurement indicators}

A postoperative SCT scan was performed to evaluate the accuracy of the mandibular reconstruction. Through the comparison between preoperative design and postoperative SCT 3D reconstruction model images, different colors were used to distinguish the distance between the corresponding points on the superimposed images, and the maximum deviation value was counted. The deviation of the fibular segment on the SCT model and the distance of the deviation between the mandibular angle and condylar outer pole were calculated to evaluate the surgical accuracy.

\section{Statistical analysis}

The data were collected from three separate experiments and expressed as means standard deviation, and a comparative t-test was applied for statistical analysis by SPSS 22.0 
Table 1 Dimension change of the PLA guide plates with different filling rates after high temperature sterilization (mm)

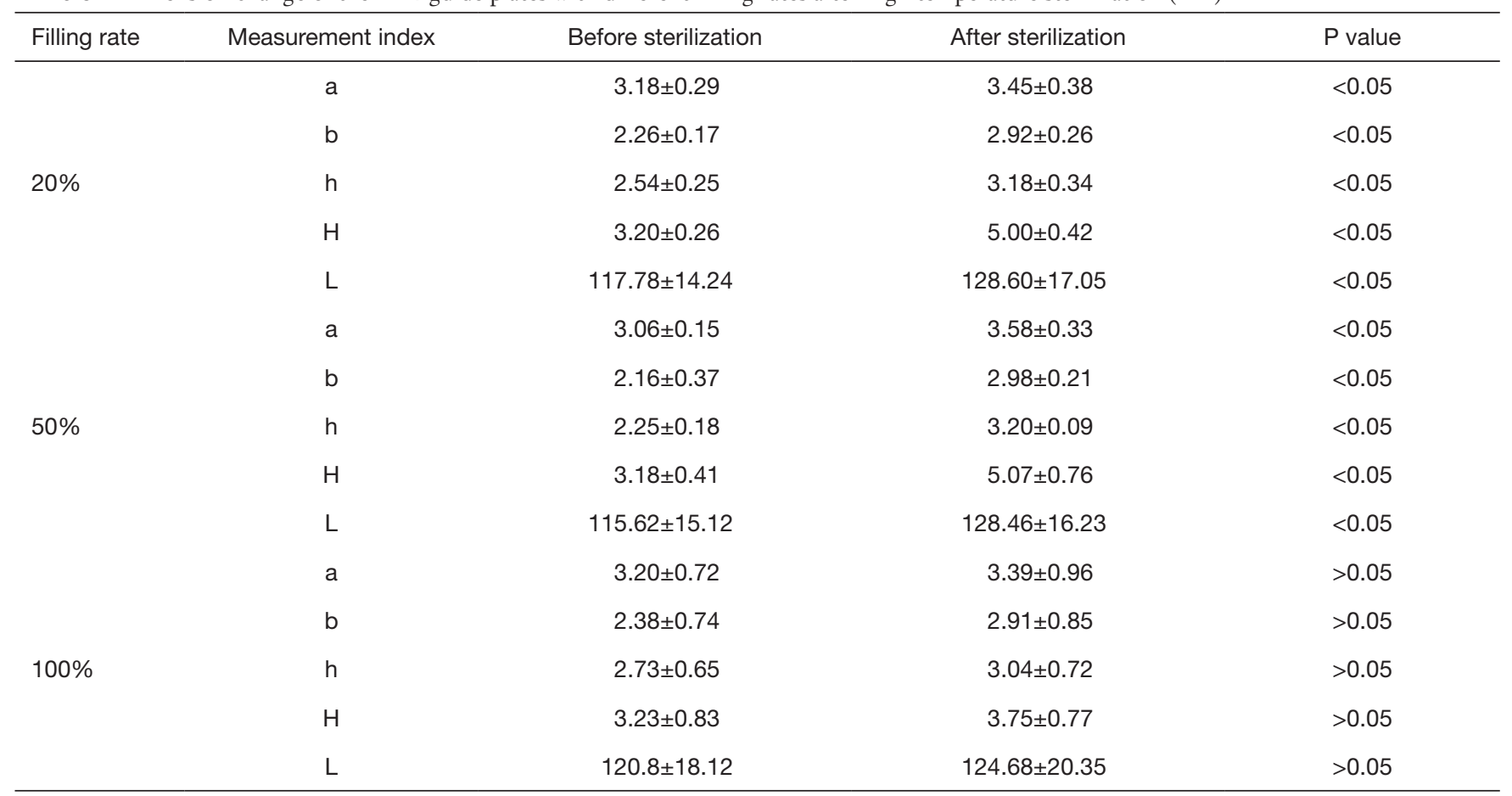

a,b, thickness at both ends of the samples; $h$, thickness of the midpoint of the long side of the samples; $L$, length of the long side of the samples; $\mathrm{H}$, vertical height between the long side midpoint and level table of the samples; $\mathrm{K}$, curvature of the lower midpoint of the long side of the samples after sterilization.

statistical software. A P value $<0.05$ was considered to be statistically significant.

\section{Results}

\section{Dimension change of the PLA guide plates with different filling rates after high temperature sterilization}

The deformation measurement data are shown in Table 1. The curvature of the standard PLA sample with a $50 \%$ filling rate was the largest of all, and the effect of temperature on the size deformation was the most remarkable $(\mathrm{P}<0.05)$. Meanwhile, the standard PLA with a $100 \%$ filling rate had the smallest curvature and the least significant size deformation after high-temperature sterilization $(\mathrm{P}>0.05)$. The curvature of the PLA sample was not analyzed because of the slight deformation before and after high temperature sterilization.

\section{Dimension change of the PLA guide plates with different thicknesses after high temperature sterilization}

As shown in Table 2, if the filling rate was $100 \%$, the
PLA sample with a $3.2 \mathrm{~mm}$ thickness had the maximum curvature, and its dimension most obviously distorted $(\mathrm{P}<0.05)$. When the thickness was $4.8 \mathrm{~mm}$, the PLA sample had the minimum curvature and the least obvious deformation $(\mathrm{P}>0.05)$. Because the error of repeated measurement was below $0.1 \mathrm{~mm}$, so the influence of the error in the repeated measurement on the clinical operation can be ignored. Based on the above consideration, when the filling rate was $100 \%$ and the thickness was $4.8 \mathrm{~mm}$ in this study, the deformation of the test piece after high temperature sterilization was minimal, so we thought that 3D-printing surgical guide plate should be designed with a filling rate of $100 \%$ and a thickness of $4.8 \mathrm{~mm}$.

\section{The measurement of surgical accuracy}

As shown in Table 3, three months after operation, the distance between the lateral convex point of the condyle, the medial convex point of the condyle, and the horizontal contained angle of the long axis of condyle were $128.34 \pm 8.68 \mathrm{~mm}, 88.69 \pm 6.75 \mathrm{~mm}$, and $145.87^{\circ} \pm 12.01^{\circ}$, respectively. The maximum error of the remaining mandible 
Table 2 Dimension change of the PLA guide plates with different thicknesses after high temperature sterilization

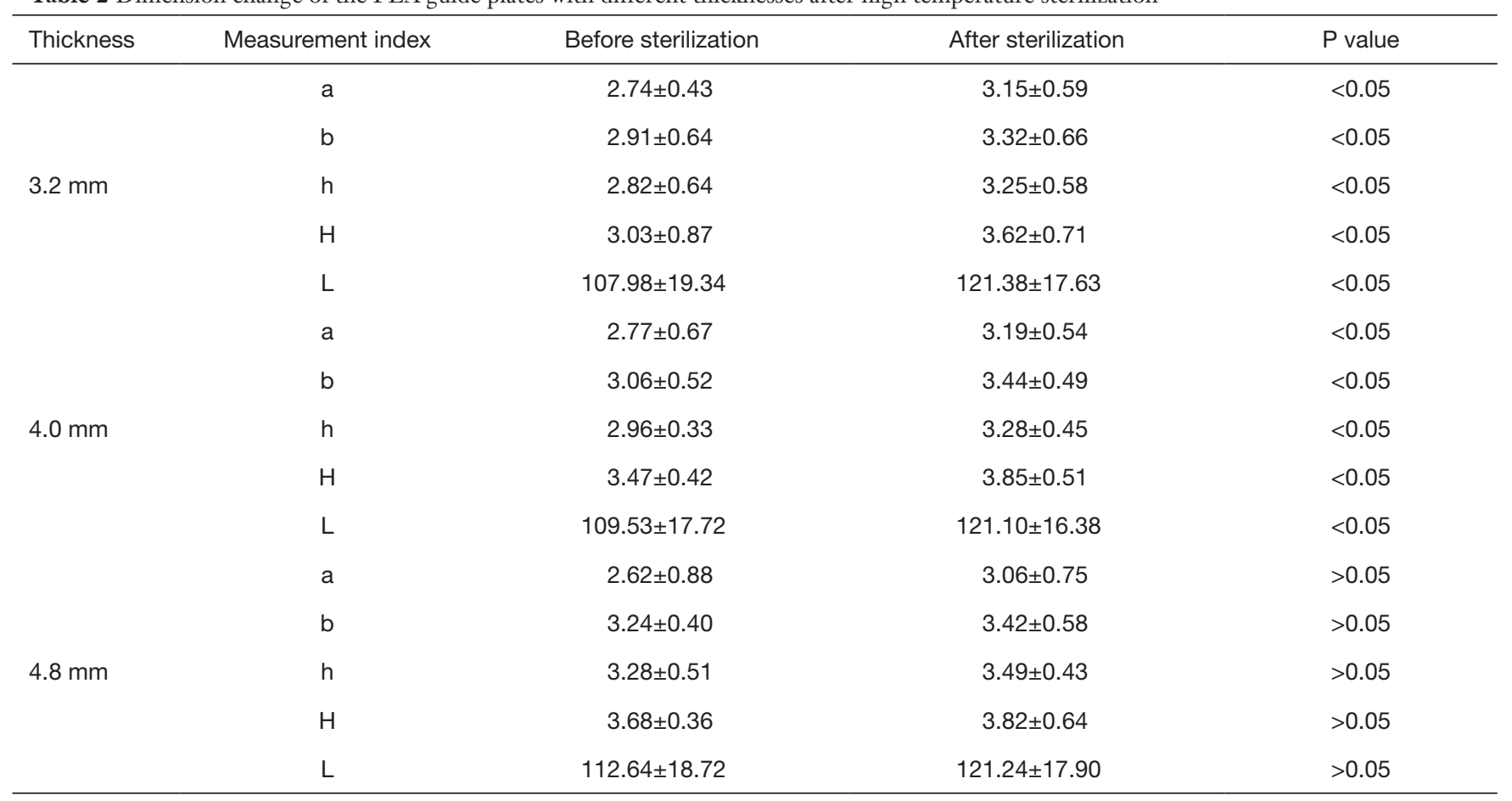

All samples had a filling ratio of $100 \%$.

after registration was $1.67 \pm 0.63 \mathrm{~mm}$, which was a high fit. Compared with the simulated operation, the difference was not statistically significant $(\mathrm{P}>0.05)$.

\section{Clinical efficacy evaluation}

As evident from the typical cases, the operation was completed according to the preoperative design plan, and there was no serious inconsistency with the preoperative design. The incision healed well at first, and all the grafted fibular survived. The patient's face appearance was symmetrical and consistent with the expected result. The mouth opening was improved, with good recovery of the occlusal relation; there was no significant abnormality in language function or masticatory function, and the postoperative effects were within the grade I-II standard.

\section{Typical cases}

The typical case involved a male patient diagnosed with squamous cell carcinoma of the left mandibular gingiva. The surgical design included partial mandibulectomy, extended resection of the gingival carcinoma, neck lymphoidectomy, and simultaneous reconstruction the mandible with a fibular flap graft. The osteotomy scope, surgical guide plate, and postoperative 3D model (Figure 4) were imported into the open-source software, NetFABb, in STL format for printing and support design. It is worth noting that the non-working face was used for the support, and the working face was as smooth as possible. After the direction of the STL files were adjusted, followed by importation into opensource software, KISSlicer, for section design. With the $100 \%$ filling rate of the guide plate and the $20 \%$ filling rate of the postoperative $3 \mathrm{D}$ model, the printing document was imported into the FDM 3D printer in GRCODE format to generate the model of the guide plate and reconstructed mandible (Figure 5). Then, the titanium plate was performed on the postoperative $3 \mathrm{D}$ model, and was fixed by a titanium screw on the mandible model. After the titanium plate and screws were removed, the PLA surgical guide plate was placed and fixed on the postoperative $3 \mathrm{D}$ model by titanium screws, with the screw holes on the plate exactly corresponding to those on the model (Figure 6). This design method omits connecting the guide plate to maintain the relative position of the remaining mandible after osteotomy and shortens the operation process.

During the operation, the osteotomy guide plate was accurately placed on the mandible according to the 
Table 3 Three-dimension registration of mandibular CT before and after operation

\begin{tabular}{lcc}
\hline Measurement index & Preoperative & Postoperative \\
\hline Distance between the lateral convex points of the condyles $(\mathrm{mm})$ & $126.21 \pm 8.34$ & $128.34 \pm 8.68$ \\
Distance between the interior convex points of the condyles $(\mathrm{mm})$ & $87.16 \pm 6.52$ & $88.69 \pm 6.75$ \\
Horizontal contained angle of the long axis of the condyle $\left(^{\circ}\right)$ & $143.40 \pm 11.56$ & $145.87 \pm 12.01$ \\
Maximum deviation after registration $(\mathrm{mm})$ & $1.25 \pm 0.58$ & $>0.05$ \\
\hline
\end{tabular}
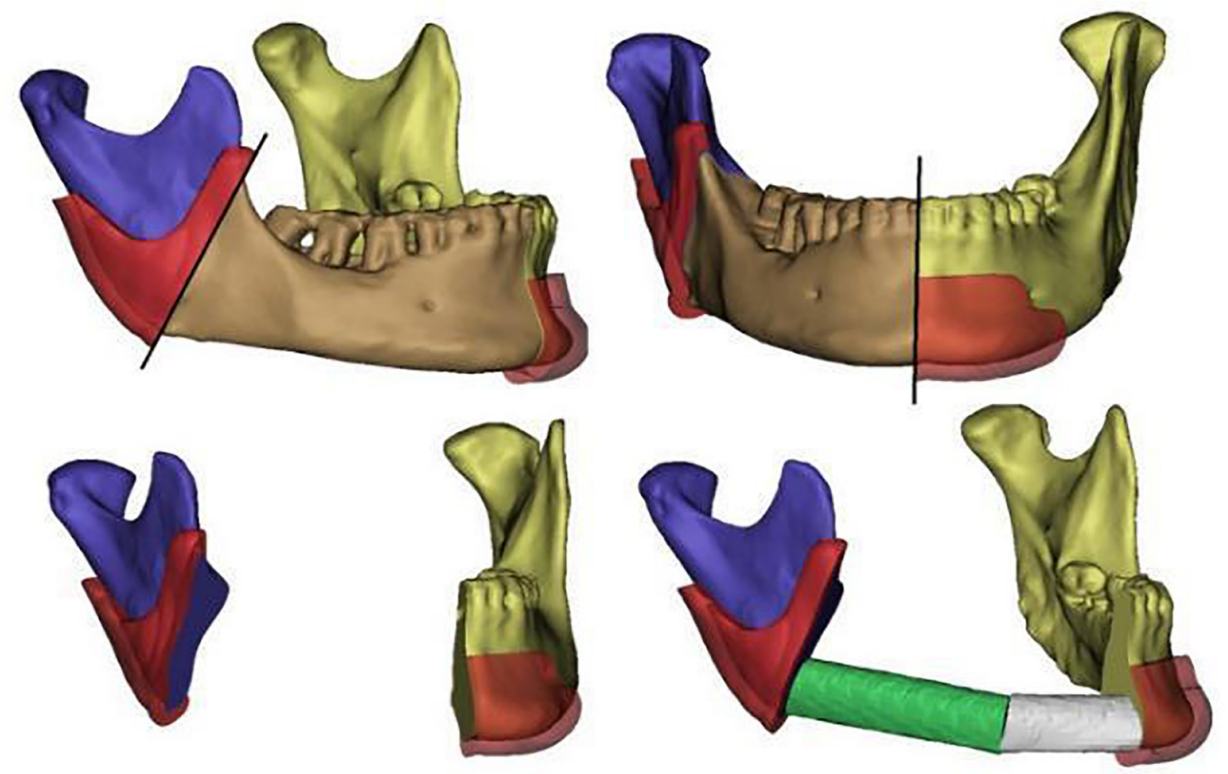

Figure 4 The design of the ostectomy guide plate.

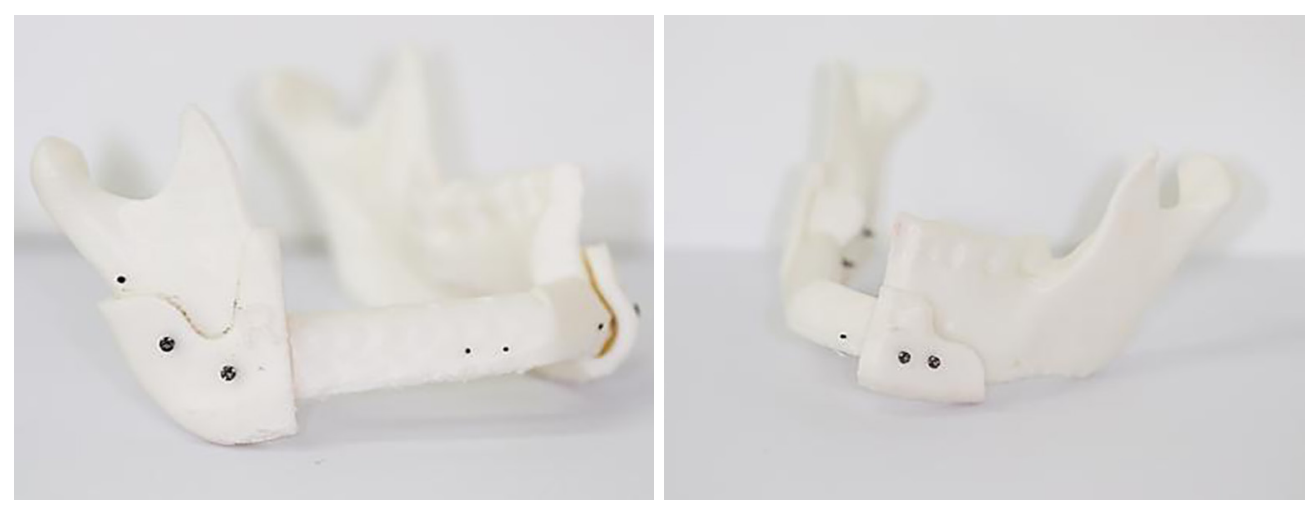

Figure 5 3D-printed fibula reconstructed mandible and osteotomy guide placement diagram.

preoperative design, and holes were drilled on the mandible under guidance of a screw hole on the guide plate. After the guide plate was fixed on the mandible with titanium screws, both ends of the mandibular lesions were respectively cut off along the osteotomy edge of the guide plate (Figure 7). Then, the titanium screw and guide plate were removed, and the preformed titanium plate was aligned with the corresponding screw holes on the remaining mandible 

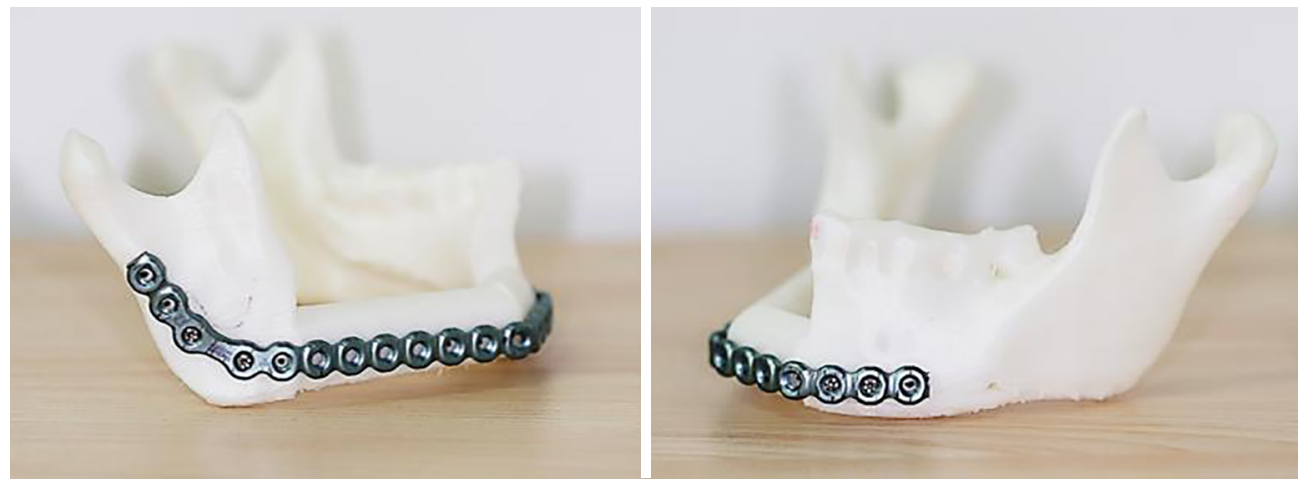

Figure 6 The matching between the screw hole of the preformed titanium plate and the 3D printing PLA guide plate.

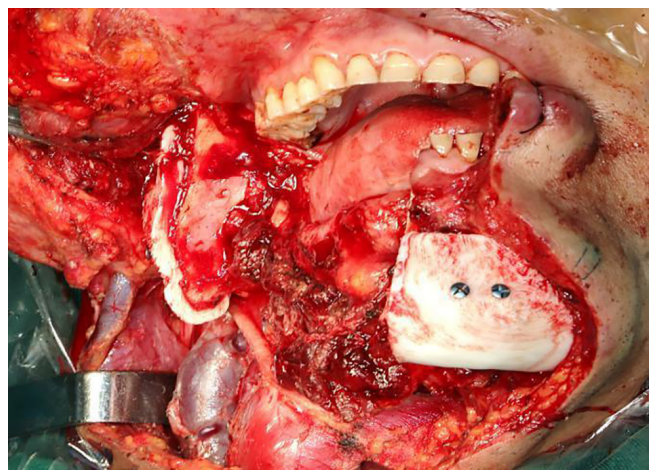

Figure 7 Titanium screw of the guide plate fixation on the mandible and amputation lesion area along the edge of the guide plate.

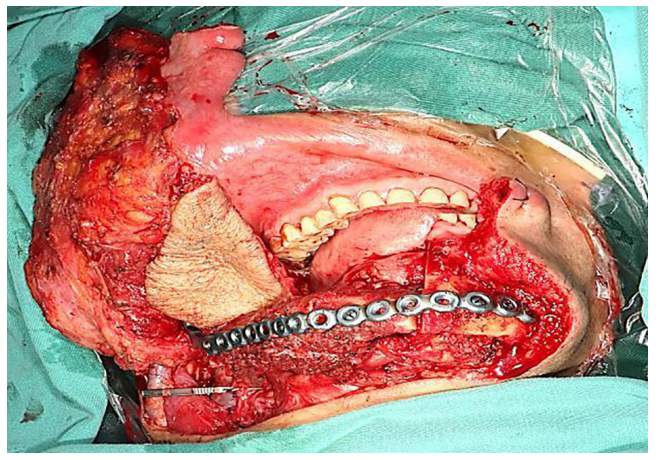

Figure 8 Implantation of the preformed titanium plate following scheduled hole screwing and fixation of the fibular segment.

according to the preoperative design. The titanium plate was fixed with screws, and the normal occlusal relation of the remaining teeth was confirmed (Figure 8).

Postoperative SCT examination was performed to reconstruct the $3 \mathrm{D}$ image of the mandible, and the data was fitted and compared with the preoperative design. The results showed that the actual reconstruction effect was basically consistent with that of the preoperative design scheme and that the occlusal relation of the remaining teeth of the patient was normal (Figure 9).

The second typical case was a female patient diagnosed with ameloblastoma of the right mandible. The surgical design was a partial resection of the right mandible and simultaneous reconstruction of the mandible with a left fibular flap. The range of osteotomy, the position of the osteotomy guide and postoperative $3 \mathrm{D}$ model design are shown in (Figure 10), which is similar to the first case above. A preformed titanium plate was made on the postoperative $3 \mathrm{D}$ printing model, and the screw holes between the PLA surgical guide plate and titanium plate were an exact fit (Figure 11). The osteotomy guide plate and the preformed titanium plate were separately sterilized by high-temperature steam before being used for subsequent experiments.

According to the preoperative design, the anterior edge of the right mandibular lesion was cut off by the osteotomy guide, and the mandible condyle neck was cut obliquely during the operation, but the posterior margin of the condyle was too small to install the osteotomy guide (Figure 12). After the titanium screws and the guide plate were removed, the preformed titanium plate was used to fix the shape of the fibular flap, with the screw holes between the PLA surgical guide plate and titanium plate being exactly matched. When the mandibular condyle was in the retruded contact position, the mandible condyle neck was fixed to the distal end of the grafted fibular with a wire, and then the occlusal relation of the remaining teeth was checked.

After the operation, maxillofacial SCT examination showed 

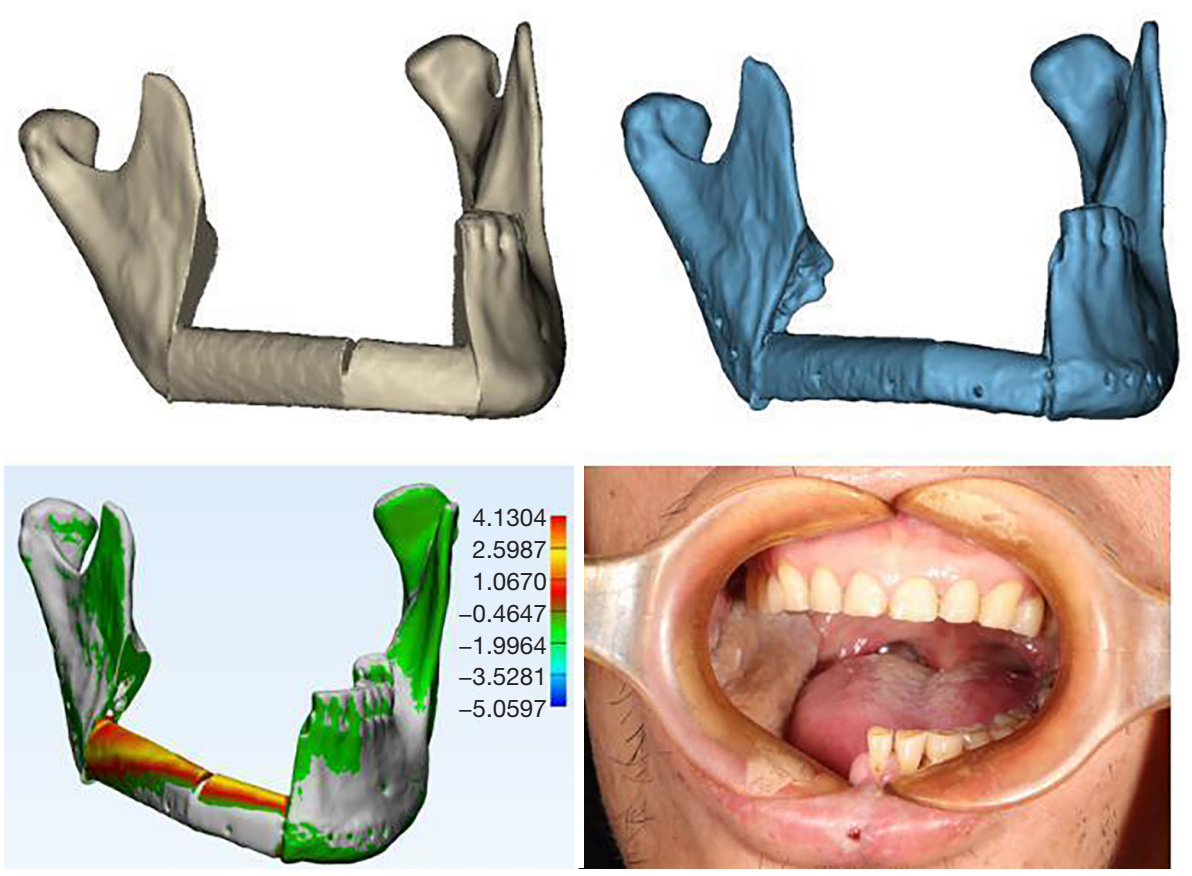

Figure $93 \mathrm{D}$ registration of the preoperative design and postoperative reconstruction.
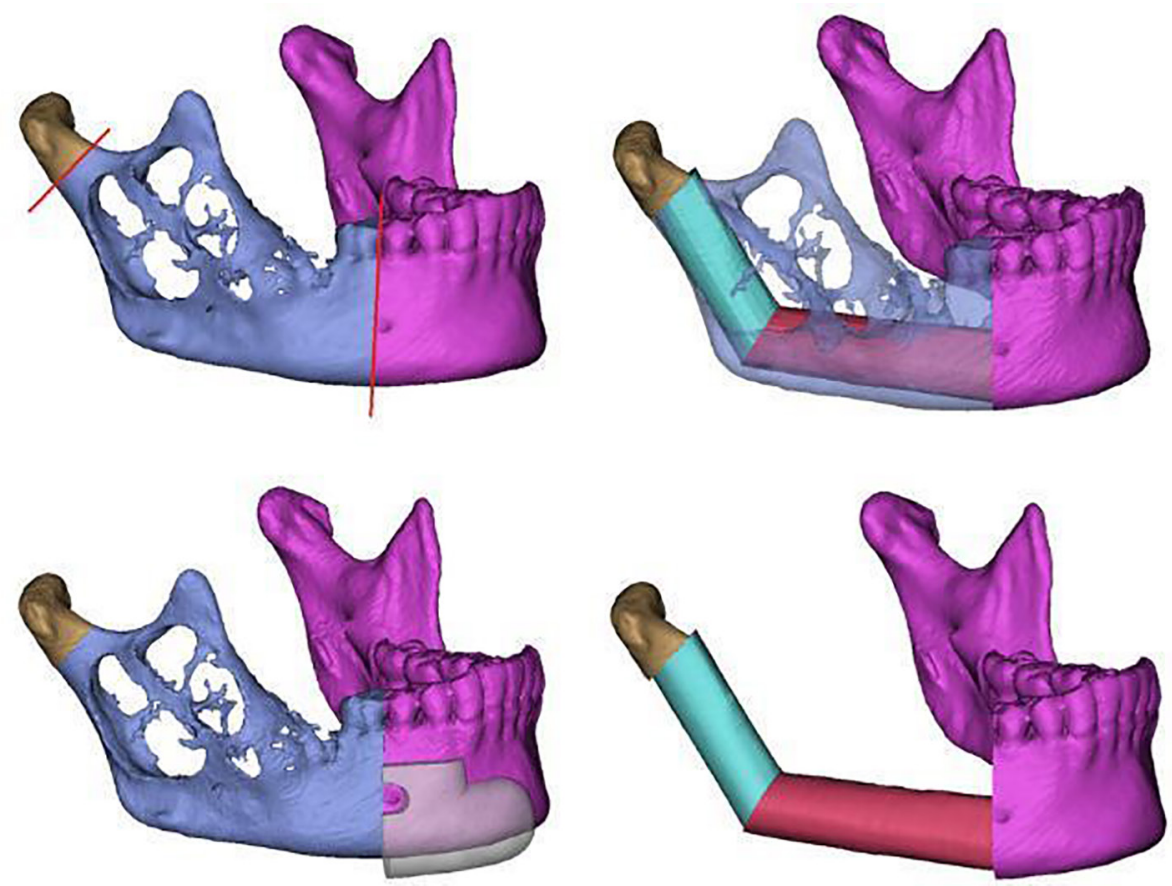

Figure 10 The digital design of mandibular resection range, osteotomy guide design and the reconstructed mandible. 


\section{Page 10 of 14}

that the postoperative effect of mandible reconstruction was basically the same as that of the preoperative design, and the occlusal relation of remaining dentition was normal, with any errors mainly concentrated on the lateral part of the right condyle (Figure 13).

In all, 18 cases underwent a similar method of partial mandibular resection and simultaneous reconstruction with a fibular graft. After the operation, the occlusal relation of the remaining teeth was basically the same as that before the operation, and there was no obvious difference in mastication, pronunciation, or temporomandibular discomfort. In order to evaluate the accuracy of this method, the distance between the bilateral condyles and the horizontal angle of the long axis of the bilateral condyles were measured before and after the operation (Figure 14). Through matching of the preoperative design and the $3 \mathrm{D}$ CT model of the mandible 1 month after operation, it was found that the maximum deviation value of the remaining mandible after fitting was not significantly different before and after the operation.

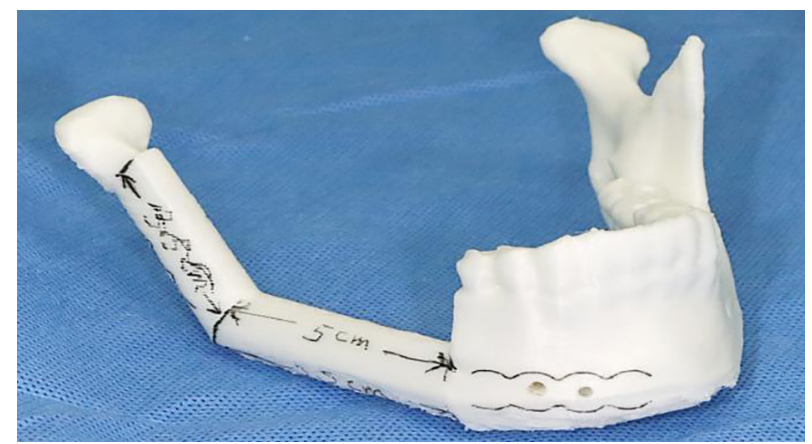

Figure 11 Simulation of the postoperative mandible and determination the retention hole of the $3 \mathrm{D}$ guide plate.
Wu et al. 3D printing PLA guide plate in mandible reconstruction

\section{Discussion}

The goal of mandibular defect reconstruction is to both achieve functional recovery and a good facial aesthetic. There are many important factors involved in this procedure, including the particular shapes and complex movement form, the types of lesions, and the location and extent of defect $(10,11)$ which can influence the clinical effect of mandibular reconstruction. Vascularized free fibular graft is currently the most prevalent method for repairing defect of the mandible (12). For better functionality and aesthetic effect, the fibula usually needs to be osteotomized and remodeled. However, data acquisition and operative program design in the traditional surgical procedure depends only on the actual model and the 2D image, and it is thus difficult to achieve an accurate reconstruction, because the adjustment of transplanted bone blocks and mandibular contour rely heavily on the experience and skill of the surgeon. These limitations result in an unsatisfactory restorative effect in the traditional surgical mode. For large defects of the mandibular ramus and the anterior part of the mandibular body, the reconstruction of the individual anatomical morphology and masticatory function is particularly challenging.

In recent years, digital surgical technology has played an increasingly important role in personalized and accurate mandibular reconstruction. Compared with the traditional surgical model that relies excessively on experience, digital surgical technology greatly improves the individualization, accuracy, and safety of the procedure (13). Previous evidence has shown that the application of digital surgical technology can significantly improve the clinical effect of mandibular defect reconstruction $(14,15)$. There are several advantages in digital surgical technology: (I) the $3 \mathrm{D}$ reconstruction
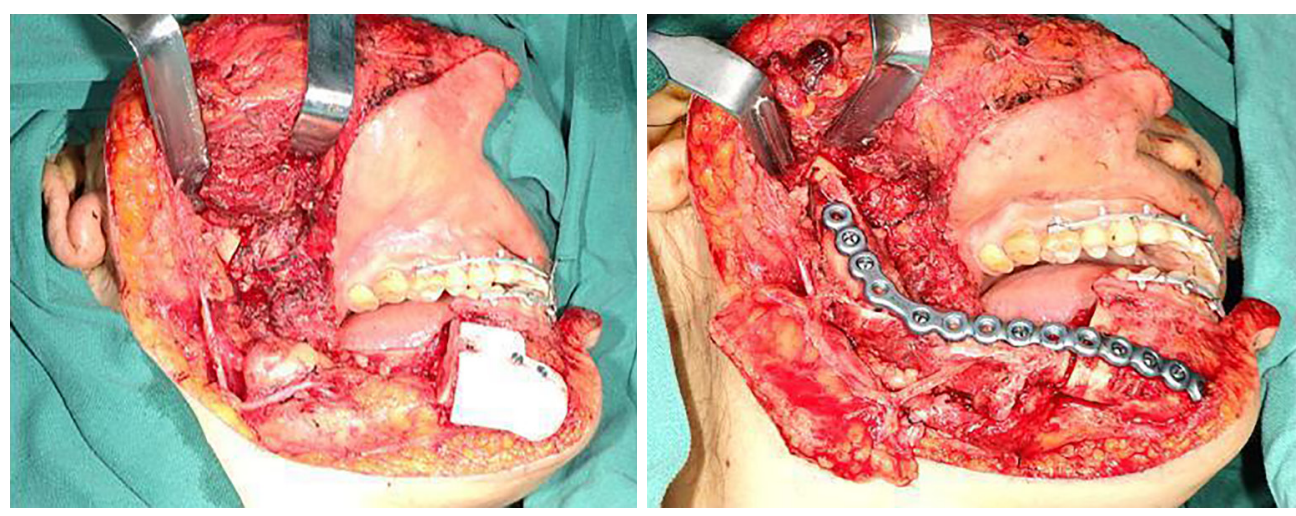

Figure 12 Intraoperative osteotomy guided by the 3D-printeed guide plate and mandibular reconstruct with fibula graft. 

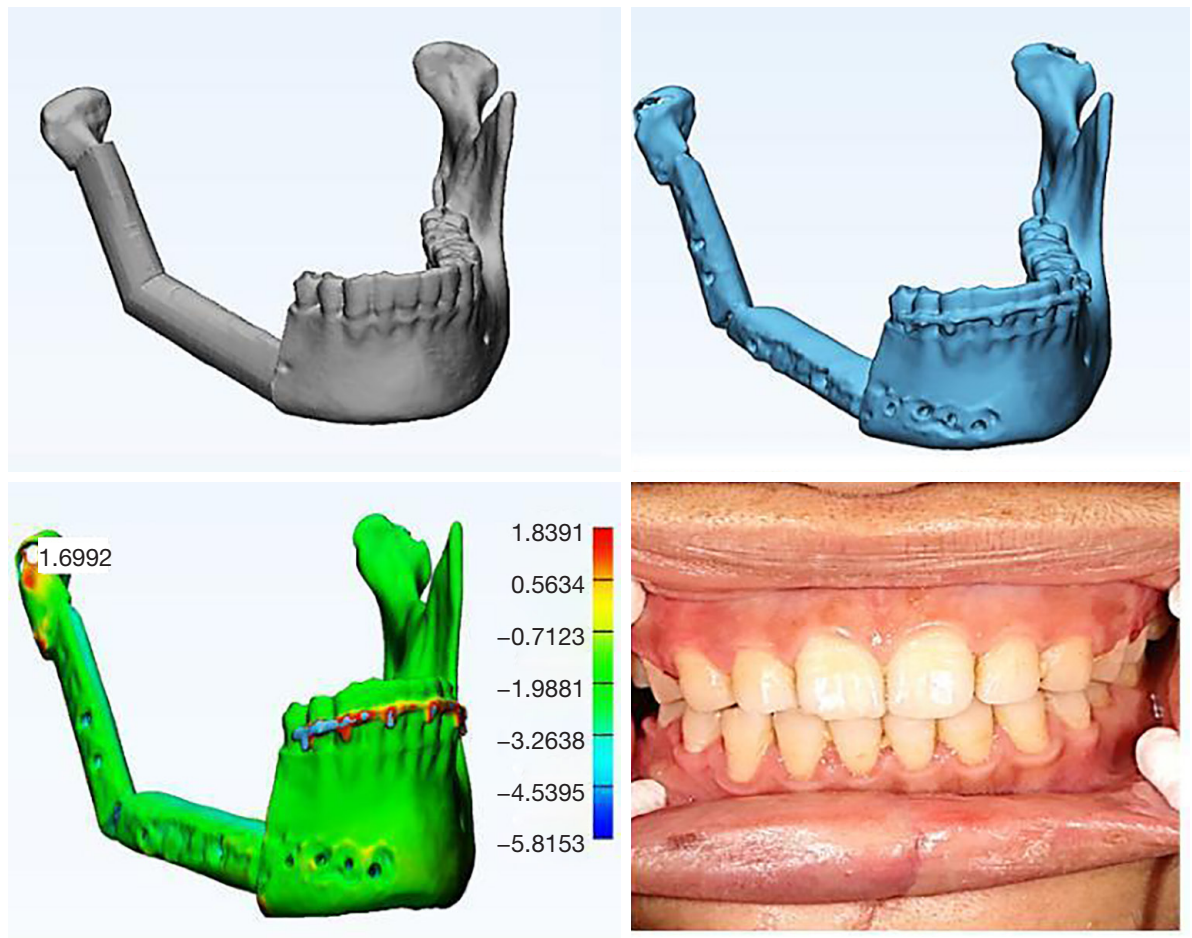

Figure 13 The registration of postoperative 3D reconstruction and simulation design.
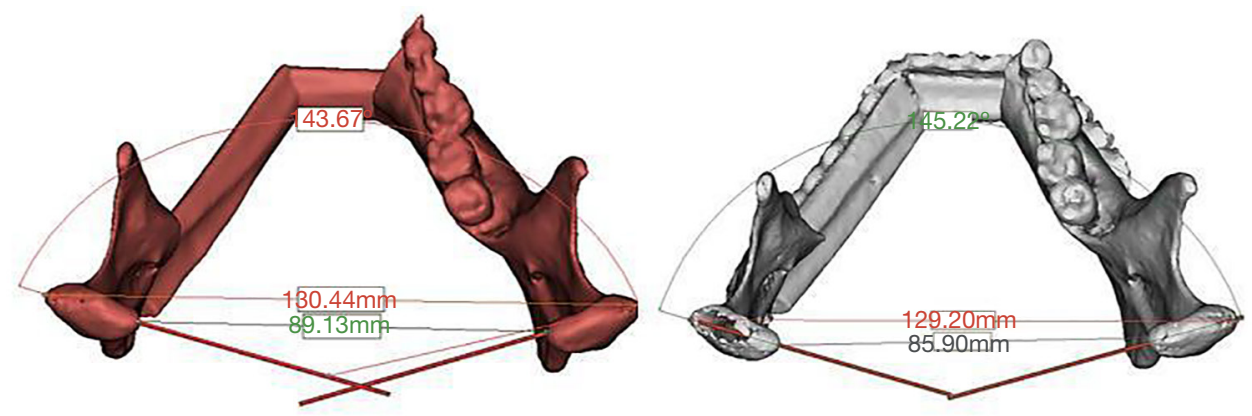

Figure 14 The measurement of accuracy before and after operation.

technique can intuitively reflect the state of the mandibular defect and its surrounding tissues; (II) computer-aided technology can build an individualized model and improve the treatment plan in the simulation operation; (III) 3D printing technology can be used to prepare a surgical guide plate to ensure the accurate guidance and positioning of the surgery.

With the gradual development of the $3 \mathrm{D}$ printing technology, a widening array of personalized bioprinting materials has been applied in the medical field and achieved satisfactory clinical utility (16). As a striking example of digital technology being used in biomaterials, the $3 \mathrm{D}$-printed surgical guide plate has been proven to be an effective measure in realizing individualized precision for surgical operation (17). It further ensures the accurate implementation of the surgery according to a preoperative plan and reduces the difficulty of operation through simplification.

There are many kinds of $3 \mathrm{D}$-printed materials, and their physicochemical performance and corresponding processing methods also vary; these include FDM technology for processing acrylonitrile butadiene styrene (ABS) 
resin, stereolithography apparatus (SLA) technology for photosensitive resin, 3D printing technology for gypsum powder, laser sintering technology for metal powder, and others (18). The mechanical and bioactive properties of the $3 \mathrm{D}$-printed surgical guides thus each have their own strengths and weaknesses. Some scholars have found that a 3D-printed guide plate composed of resin or metallic material can accurately restore preoperative design in the operation and successfully achieve mandibular defect reconstruction in an individualized fashion $(19,20)$. Unfortunately, 3D-printed materials and the supporting equipment are expensive, and the maintenance cost is also high.

Polylactic acid is relatively biocompatible, thermostable, inexpensive, and widely applied as guide material for medical implants, especially for FDM consumables (21). In addition, PLA has good strength and a melting point of $170-230{ }^{\circ} \mathrm{C}$, which is suitable for use as an osteotomy guide plate with mainstream FDM technology (22). This method has advantages of simple operation and fast forming speed, with an average duration of 3 hours. In terms of printing precision, the postoperative CT scanning in the present study showed that the deviation of distance between the lateral convex point of the condyle was $2.13 \pm 0.34 \mathrm{~mm}$, the deviation of distance between the medial convex point of the condyle was $1.53 \pm 0.23 \mathrm{~mm}$, and the mean variation of the horizontal angle of the long axis of the condyle was $2.47^{\circ} \pm 0.45^{\circ}$; thus osteotomy using a $3 \mathrm{D}$-printed PLA guide plate was accurate and met the clinical needs of patients.

It was reported that sterilization by Co60 irradiation causes structural the damage and mechanical change of PLA material, while ethylene oxide sterilization has no significant effect (23). Furthermore, the heat resistance of new PLA material has been greatly improved and can meet the requirements of high-temperature sterilization. We measured the smaller deformation of PLA with different filling rates after sterilization, and found that high-pressure steam sterilization could ensure the accuracy of the PLA guide plate.

In this study, the $3 \mathrm{D}$-printed surgical plate based on PLA material was used to guide the reconstruction of mandibular segmental defect with a fibular graft. The guide plate was designed to fix on the remaining teeth and reduce normal mandibular bone surface and as small as possible. According to the preoperative design, the typical anatomical structure of the remaining jaw for fixing the guide were determined, and the guide could be accurately placed on the jaw immediately after the lesion was exposed during the operation. In all 18 cases, the 3D-printed PLA guide plate was placed smoothly and attached to the bone surface, while the lesion range was consistent with the preoperative simulation, which ensured the stability of the intraoperative guide plate. To increase the retention of the surgical guide plate, a reservation design for the mucosa, mental foramen, and other structures was made on the basis of $3 \mathrm{D}$ bone reconstruction in virtual surgery to intraoperatively prevent disturbances in the soft tissue.

The research results showed that the digital design plan could be precisely be duplicated into the operation process through the $3 \mathrm{D}$-printed PLA guide plate. The operation time of the mandibular reconstruction was significantly shortened, the intraoperative blood loss was reduced, and postoperative perforation, numbness, pain or looseness of the mandible were not observed, which is similar to the results of relevant studies (24). Ischemic time is considered to be an important factor affecting the survival rate of the fibular grafts, but computer-aided modeling or computeraided machining (CAD/CAM) technology has obvious advantages over traditional surgical methods. According to the jaw osteotomy guide designed by our research, the results of all the operations performed show that, the postoperative measurement of mean error between the preoperative virtual-designed osteotomy and the actual surgical osteotomy was $1.67 \pm 0.63 \mathrm{~mm}$, suggesting the removed bone to be highly matched with the $3 \mathrm{D}$-printed PLA guide plate. These results were basically consistent those of other recent research, which also reports that use of a 3D-printed PLA guide plate has satisfactory accuracy and high clinical value (25).

The clinical evaluation, including postoperative facial appearance, occlusal stability, mouth opening and chewing, and phonation function of the patients all achieved a grade I-II standard. The higher degree of satisfaction further confirmed the surgical effect, which may be related to the fact that digital technology can ensure the consistency of space and morphology of the original jawbone (26). Digital design combined with 3D-printing-assisted surgery can not only significantly improve the surgical effect, but also better meet the desired aesthetic effect in the jaw bone, and thus improve the overall satisfaction that patients have with the surgery. Meanwhile, computer-aided design combined with $3 \mathrm{D}$ printing technology can more accurately reflect the patient's lesion area and jaw bone.

As indicated above, the 3D-printed digital PLA guide plate has a high osteotomy accuracy and contributes significantly to improving the clinical effect of fibular flap grafting for reconstruction of mandibular defects. It can 
not only shorten the operation time, but also reduce the operation risk. The application of the $3 \mathrm{D}$-printed PLA guide plate may provide a basis for the accurate formulation of a treatment plan, has high clinical value, and thus warrants further promotion. However, the long-term results of the facial shape, occlusal relation, and joint movement recovery of patients still need to be verified by the inclusion of a greater number of clinical cases with observation over a longer timeframe.

At present, the most widely used $3 \mathrm{D}$ printing in the biomedical field is the application of surgical guides and a small number of personalized prostheses. We believe that the ultimate goal of $3 \mathrm{D}$ printing in the medical field should be the printing of biomaterials and the production of personalized biological organs with complete physiological functions. Of course, this ideal goal requires broad biomedicine and materials science and 3D printing technology. The majority of scholars work together and cooperate, and believe that with the development of materials science and the progress of science and technology, this goal will eventually be realized.

\section{Acknowledgments}

Funding: This study was financially supported by Military Medical Science and Technology Youth Training Program (No. 18QNP038), Beijing Excellent Talents Training Project (No. 2017000062586G232) and Academy of Military Medical Sciences Innovation Fund (2017CXJJ14).

\section{Footnote}

Reporting Checklist: The authors have completed the MDAR reporting checklist. Available at http://dx.doi.org/10.21037/ atm-20-6781

Data Sharing Statement: Available at http://dx.doi. org/10.21037/atm-20-6781

Conflicts of Interest: All authors have completed the ICMJE uniform disclosure form (available at http://dx.doi. org/10.21037/atm-20-6781). The authors have no conflicts of interest to declare.

Ethical Statement: The authors are accountable for all aspects of the work in ensuring that questions related to the accuracy or integrity of any part of the work are appropriately investigated and resolved. All procedures performed in this study involving human participants were in accordance with the Declaration of Helsinki (as revised in 2013). This study was approved by the Medical Ethics Committee of the General Hospital of the Western Theater command of the PLA (No. 2018-5-15). Informed consent was obtained from all the patients.

Open Access Statement: This is an Open Access article distributed in accordance with the Creative Commons Attribution-NonCommercial-NoDerivs 4.0 International License (CC BY-NC-ND 4.0), which permits the noncommercial replication and distribution of the article with the strict proviso that no changes or edits are made and the original work is properly cited (including links to both the formal publication through the relevant DOI and the license). See: https://creativecommons.org/licenses/by-nc-nd/4.0/.

\section{References}

1. Smith BT, Shum J, Wong M, et al. Bone tissue engineering challenges in oral \& maxillofacial surgery. Adv Exp Med Biol 2015;881:57-78.

2. Löfstrand J, Nyberg M, Karlsson T. Quality of Life after free fibula flap reconstruction of segmental mandibular defects. J Reconstr Microsurg 2018;34:108-20.

3. Melville JC, Nassari NN, Hanna IA, et al. Immediate Transoral Allogeneic Bone Grafting for Large Mandibular Defects. Less Morbidity, More Bone. A Paradigm in Benign Tumor Mandibular Reconstruction? J Oral Maxillofac Surg 2017;75:828-38.

4. Kakabadze A, Mardaleishvili K, Loladze G, et al. Reconstruction of mandibular defects with autogenous bone and decellularized bovine bone grafts with freezedried bone marrow stem cell paracrine factors. Oncol Lett 2017;13:1811-8.

5. García-Mato D, Ochandiano S, Garcia-Sevilla M, et al. Craniosynostosis surgery: workflow based on virtual surgical planning, intraoperative navigation and 3D printed patientspecific guides and templates. Sci Rep 2019;9:17691.

6. Szuhanek C, Mihai AM, Sarbu A, et al. 3D Printed surgical guides used in orthodontics. Mater Plast 2019:56:657-9.

7. Yang WF, Choi WS, Leung YY, et al. Three-dimensional printing of patient-specific surgical plates in head and neck reconstruction:A prospective pilot study. Oral Oncol 2018;78:31-36

8. Meng X, Bocharova V, Tekinalp H, et al. Thoughening of nanocelluose/PLA composites via bio-epoxy interaction: mechanistic study. Materials \& Design 2018;139:188-97. 


\section{Page 14 of 14}

9. ASTM D790-2010, Standard test methods for flexural properties of unreinforced and reinfored plastics and electrical insulating materials.

10. Sales PHD, Cetira EL, Oliveira Neto JQD, et al. Rapid prototyping as an auxiliary in mandibular reconstructions. J Craniofac Surg 2017;28:e744-5.

11. Akinbami BO. Reconstruction of continuity defects of the mandible with non-vascularized bone grafts. systematic literature review. Craniomaxillofac Trauma Reconstr 2016;9:195-205.

12. Kakabadze A, Mardaleishvili K, Loladze G, et al. Reconstruction of mandibular defects with autogenous bone and decellularized bovine bone grafts with freezedried bone marrow stem cell paracrine factors. Oncol Lett 2017;13:1811-8.

13. Wu J, Sun J, Shen SG, et al. Computer-assisted navigation: its role in intraoperatively accurate mandibular reconstruction. Oral Surg Oral Med Oral Pathol Oral Radiol 2016;122:134-42.

14. De Maesschalck T, Courvoisier DS, Scolozzi P. Computerassisted versus traditional freehand technique in fibular free flap mandibular reconstruction:a morphological comparative study. Eur Arch Otorhinolaryngol 2017;274:517-26.

15. Kääriäinen $M$, Kuuskeri M, Gremoutis G, et al. Utilization of Three-dimensional computer-aided preoperative virtual planning and manufacturing in maxillary and mandibular reconstruction with a microvascular fibula flap. J Reconstr Microsurg 2016;32:137-41.

16. Pugliese L, Marconi S, Negrello E, et al. The clinical use of 3D printing in surgery. Updates Surg 2018;70:381-8.

17. Melville JC, Manis CS, Shum JW, et al. Single-unit 3D-printed titanium reconstruction plate for maxillary reconstruction: The evolution of surgical reconstruction for maxillary defects-a case report and review of current techniques. J Oral Maxil Surg 2019;77:874. e1.

Cite this article as: $\mathrm{Wu} \mathrm{P}, \mathrm{Hu} \mathrm{L}, \mathrm{Li} \mathrm{H}$, Feng L, Liu Y, Zhang S, Li XC, Zhang ML, Yang SY, Lu RJ. Clinical application and accuracy analysis of $3 \mathrm{D}$ printing guide plate based on polylactic acid in mandible reconstruction with fibula flap. Ann Transl Med 2021;9(6):460. doi: 10.21037/atm-20-6781
Wu et al. 3D printing PLA guide plate in mandible reconstruction

18. Dahake, SW, Kuthe AM, Mawale MB, et al. Applications of medical rapid prototyping assisted customized surgical guides in complex surgeries. Rapid Prototyping J 2016;22:934-46.

19. Ngo TD, Kashani A, Imbalzano G, et al. Additive manufacturing (3D printing): A review of materials, methods, applications and challenges. Compos Part B-Eng 2018;143:172-96.

20. Zhang L, Yang GJ, Johnson BN, et al. Threedimensional(3D)printed scaffold and material selection for bone repair. Acta Biomater 2019;84:16-33.

21. Sharma N, Cao SS, Msallem B. Effects of steam sterilization on $3 \mathrm{D}$ printed biocompatible resin materials for surgical guides-an accuracy assessment study. J Clin Med 2020;9:1506.

22. Xu Z, Wang NN, Liu P, et al. Poly(Dopamine) Coating on 3D-printed poly-lactic-co-glycolic acid/beta-tricalcium phosphate scaffolds for bone tissue engineering. Molecules 2019;24:4397.

23. Savaris M, dos Santos V, Brandalise RN. Influence of different sterilization processes on the properties of commercial poly(lactic acid). Mater Sci Eng C Mater Biol Appl 2016;69:661-7.

24. Kim SR, Jang S, Ahn KM, et al. Evaluation of Effective Condyle Positioning Assisted by 3D Surgical Guide in Mandibular Reconstruction Using Osteocutaneous Free Flap. Materials 2020;13:2333.

25. Lu TW, Shao Z, Liu B, et al. Recent advance in patientspecific 3D printing templates in mandibular reconstruction. J Mech Behav Biomed 2020;106:UNSP103725.

26. Jacek B, Maciej P, Tomasz P, et al. 3D printed models in mandibular reconstruction with bony free flaps. J Mater Sci Mater Med 2018;29:23.

(English Language Editor: J. Gray) 